\title{
ATLAS-BASED BRAIN EXTRACTION IS ROBUST ACROSS RAT MRI STUDIES
}

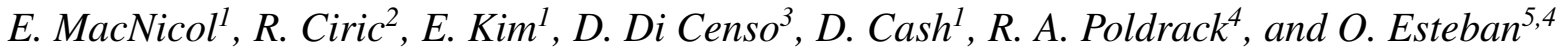 \\ ${ }^{1}$ Dept. of Neuroimaging, Institute of Psychiatry, Psychology and Neuroscience, \\ King's College London, London, UK \\ ${ }^{2}$ Dept. of Bioengineering, Stanford University, Stanford, CA, USA \\ ${ }^{3}$ Dept. of Life, Health and Environmental Sciences, University of L'Aquila, L'Aquila, Italy \\ ${ }^{4}$ Dept. of Psychology, Stanford University, Stanford, CA, USA \\ ${ }^{5}$ Dept. of Radiology, Lausanne University Hospital and University of Lausanne, Switzerland
}

\begin{abstract}
Brain extraction is a ubiquitous initial step in MRI analysis pipelines. Although there are several solutions for human imaging, existing rodent imaging tools often perform poorly on new data with acquisition protocols and parameters that differ from the images with which the tools were developed. Here, we adapted antsBrainExtraction (from the popular ANTs neuroimaging toolbox), which implements atlasbased brain extraction of adult humans, for use with rat MRI data. The new tool, artsBrainExtraction, was evaluated on 190 images from three different scanners, utilising six imaging protocols that differ in contrast, resolution, and field-ofview. The tool produced highly accurate brain masks in 186 (98\%) of the images and adequate masks in the other four. We present a reliable brain extraction tool for rodent images that is agnostic to the imaging protocol and can be extended to further image modalities and non-human populations.
\end{abstract}

Index Terms - brain extraction, preclinical MRI, rodent, MRI, preprocessing

Acknowledgments EM is supported by the UK Medical Research Council and King's College London Doctoral Training Partnership in Biomedical Sciences (MR/N013700/1). $\mathrm{OE}$ is supported by the Swiss National Science Foundation, Ambizione grant PZ00P2_185872. This work was supported by the Laura and John Arnold Foundation, the NIH (NBIB R01EB020740), NIMH (R24MH114705 and R24MH117179), the Alzheimer's Society (AS-PhD-18B015), and the UK Biotechnology and Biological Sciences Research Council (BB/N009088/1). The authors have no conflicts of interests to disclose.

Compliance with ethical standards King's College London experiments were conducted in accordance with the UK Animals (Scientific Procedures) Act, 1986 and were approved by the university ethics committee. Open data were retrieved from OpenNeuro.org, released under a CC0 license.

\section{INTRODUCTION}

Brain extraction is the process of masking non-brain tissue in an image, and it is an essential preprocessing step of any neuroimaging workflow. Brain extraction improves image registration and reduces the likelihood of including signal of non-interest, which would introduce subject-wise variability. Although the problem has been reliably solved in many implementations for human MRI data (e.g., [1, 2]), these tools typically rely on prior information (e.g., shape models, probability maps, etc.) that is inappropriate for rodents and other non-human subjects. Alternatively, researchers can manually delineate the brain mask for each specimen, which is a time-consuming process subject to high inter-operator variability. Moreover, study sizes in MRI are rapidly increasing over time, ruling out manual segmentation attempts.

Automated tools increase reproducibility and reduce human error, but the existing neuroimaging software almost always expects human brains as default. This is problematic for rodent data, which differ in anatomy and various aspects of MRI acquisition. Brain extraction tools made specifically for rodent imaging have attempted to address these issues [3-8]. These methods mostly depend on the shape of the brain as defined by the image contrast, so their success is constrained to images acquired with standard parameters and with uniform signal. Thus, they can perform poorly across datasets, as a result of the idiosyncratic nature of preclinical images with respect to the MR system used and the purpose of the study, since these factors determine the image resolution, contrast, and field-of-view (FOV). However, there is an element of rodent imaging that is common across protocols and contrasts: the shape of the brain is largely homogeneous between subjects. Atlas-based methods utilize population averages and perform robustly in human imaging [9], notably using the antsBrainExtraction. sh workflow which is based on ANTs [10]). This approach is not commonly implemented in rodent pipelines, because of the paradoxical requirement of aligning an image to a template to obtain a mask, while re- 


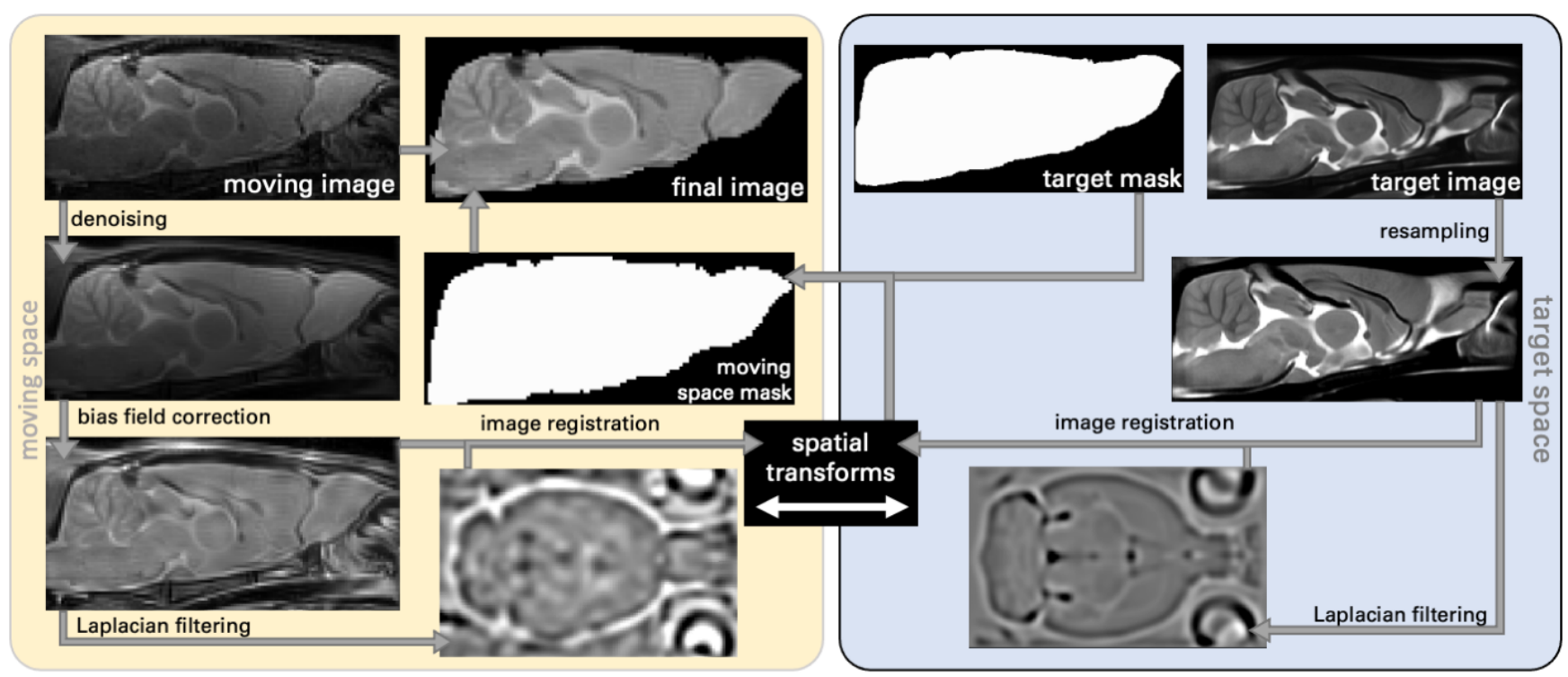

Fig. 1. The default artsBrainExtraction workflow.

quiring a mask to achieve the best alignment results. It was successfully applied in six mice by atlasBREX [6], but this implementation required a manually delineated group template and had high computational demand.

The present paper presents artsBrainExtraction (where arts stands for ANTs-based Rodents ToolS); a brain masking tool which adapts the antsBrainExtraction. sh workflow specifically to rodent imaging. artsBrainExtraction is shown to reliably implement brain extraction across rat MR data that differs by resolution, contrast variability, and FOV.

\section{MATERIALS AND METHODS}

\subsection{Data}

A multi-study dataset ( $N=190$ animals) with variable MR protocols and acquisition parameters was aggregated by combining in-house acquisitions and publicly available data.

\subsubsection{King's College London datasets}

Animals All animals were adult male rats, unless otherwise stated. Study A and B consisted of 12 and 10 Sprague-Dawley rats, respectively, while study $\mathrm{C}$ consisted of 12 Fischer-344 rats. Of these, six were wild-type and six (two females) were TgF344-AD transgenic rats [11], which express mutant human amyloid precursor protein and presenilin 1.

Body temperature, respiration rate, and peripheral oxygen saturation (Small Animal Instruments, Inc; Stony Brook, NY) were monitored throughout scanning. All animals were initially anaesthetised with $5 \%$ isoflurane in an 80:20 mix of medical air and oxygen. Animals in study $\mathrm{C}$ were maintained with $2-2.5 \%$ isoflurane, while animals in studies A and B were maintained with a $65 \mathrm{mg} \mathrm{kg}^{-1}$ bolus of $\alpha$-chloralose via a tail vein cannula before infusion at a rate of $30 \mathrm{mg} \mathrm{kg}^{-1}$ hour $^{-1}$.

Scanning Parameters Data were acquired using a $9.4 \mathrm{~T}$ Bruker Biospec MR scanner with $86 \mathrm{~mm}$ volume and four channel array receiver coils. Study A acquired images with a 2D $\mathrm{T}_{2}$-weighted (T2w) RARE pulse sequence with effective TE $42 \mathrm{~ms}$; TR $1800 \mathrm{~ms}$; RARE factor $8 ; \alpha 90^{\circ}$; matrix $192 \times 128$ with $0.125 \mathrm{~mm}$ isotropic in-plane resolution; 400.7 $\mathrm{mm}$ slices, with a slice gap of $0.2 \mathrm{~mm}$, were acquired. Study B acquired images with a 3D T2w RARE pulse sequence with effective TE $42 \mathrm{~ms}$; TR $1800 \mathrm{~ms}$; RARE factor 8; $\alpha 90^{\circ}$; matrix $80 \times 120 \times 72$, with $0.25 \mathrm{~mm}$ isotropic voxels. Finally, study $\mathrm{C}$ acquired images with a $3 \mathrm{D}$ multi-gradient echo $\mathrm{T}_{2}{ }^{*} \mathrm{~W}$ pulse sequence with TE 2.7, 4.9, 7.1, 9.3, $11.5 \mathrm{~ms}$; TR $25 \mathrm{~ms}$; $\alpha 7^{\circ}$; matrix $150 \times 150 \times 100$ with $0.2 \mathrm{~mm}$ isotropic voxels.

\subsubsection{Open data}

The CAMRI dataset (Accession Number ds002870, version 1.0.0, [8]) combines various studies of T2w RARE images from 94 Sprague Dawley, 22 Long-Evans, and 16 Wistar male rats. Although the acquisition parameters are not available, the image resolution for 69 rats was $0.1 \times 0.1 \times 1 \mathrm{~mm}$ with a $256 \times 256 \times 12$ matrix, covering a limited FOV that excludes the olfactory bulb and cerebellum. All other images had 0.2 $\mathrm{mm}$ isotropic resolution with a $144 \times 144 \times 64$ matrix covering the whole brain. The TR for both resolutions was $1800 \mathrm{~ms}$.

TurboRARE T2w images from Sirmpilatze et al. (Accession Number ds001981, version 1.0.3, [12]) included 24 female Wistar rats with effective TE 33ms; TR $5225 \mathrm{~ms}$; RARE factor 8; matrix $256 \times 256$ with $0.137 \mathrm{~mm}$ isotropic in-plane resolution and 30 to $500.5 \mathrm{~mm}$ slices. 


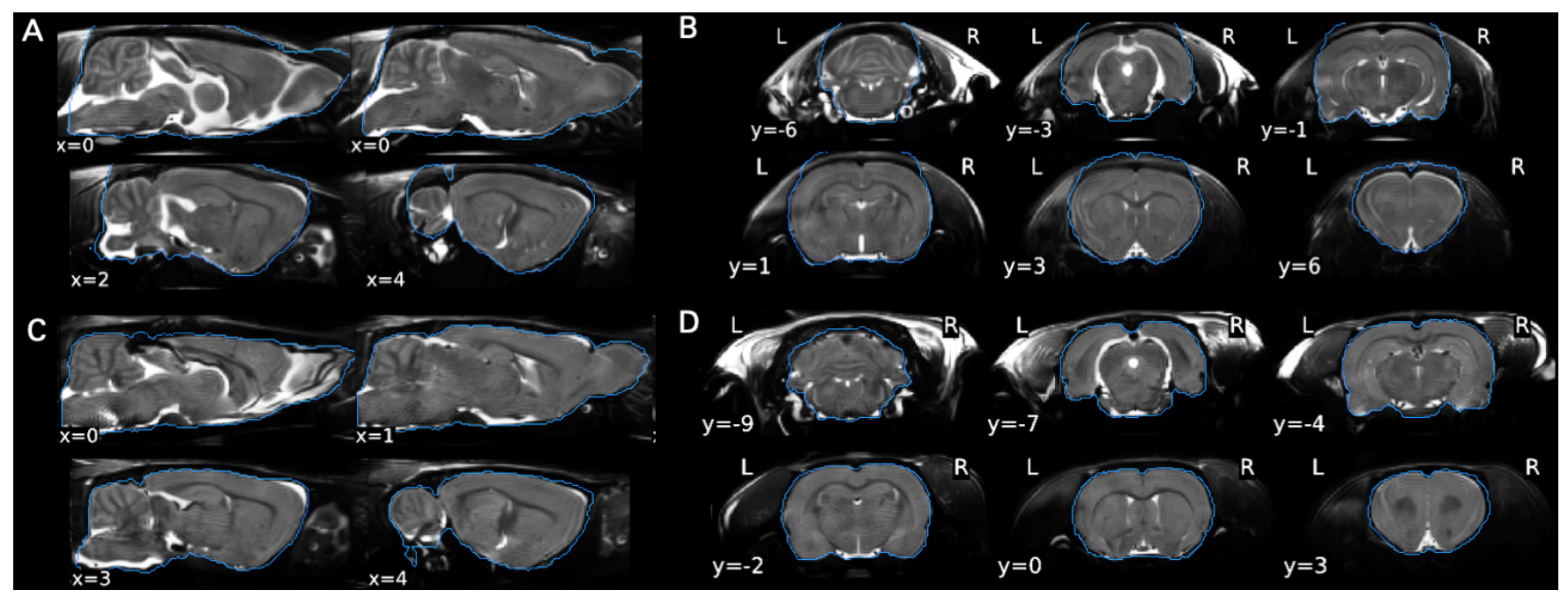

Fig. 2. Report excerpts for failed (A-B) and successful (C-D) brain extraction, with contoured mask projection.

\subsection{Atlas-based brain extraction}

Atlas-based brain extraction fundamentally relies on a precise spatial mapping between the individual specimen and a reference population-average (or template), in which a precise delineation (binary or probabilistic) of the brain is defined. The estimation of the spatial mapping between individual (moving image) and template (target image) is typically resolved with a nonlinear image registration framework. Once the mapping is estimated, the brain mask (or probability map) defined on the template space can then be projected into the individual's image space, effectively masking out the non-brain tissue.

The antsBrainExtraction.sh workflow, from which artsBrainExtraction is derived, is a mature implementation of such an approach, using an MRI template derived from the scans of 20 adult humans. The workflow first manipulates the images to facilitate the convergence of the registration process: the image histogram is truncated to discard signal intensity outliers, and the images are corrected for non-uniform intensity [13]. Both images are Laplacian filtered, which demarcates rapid changes in signal such as at the brain boundary. To estimate the transformation, a three-stage, multi-modal, multi-scale registration is implemented with rigid-body, affine, and nonlinear registrations between both the bias-corrected images and the Laplacian images. Finally, the template mask is projected into the individual's space.

\subsection{Evaluation}

All images were processed using a job submitted via a command-line interface call for artsBrainExtraction with all default settings and using Singularity containers to ensure the reproducibility of the environment. On completion of the workflow, artsBrainExtraction produces a visual report to facilitate the quality assessment of results. The visualization is stored as a dynamic SVG (Scalable Vector Graphics), which can be opened with a web browser, and shows two alternating mosaic views across the axial, sagittal, and coronal planes. The first plots slices of the individual's image, while the second shows the reference rodent template resampled to the individual's image space via the inverse spatial transform. A contour denoting the brain mask edge, projected into the individual's space, is plotted on both mosaics. The contour and the smooth, fusing transition between mosaics enable evaluation of the final brain mask output and estimated spatial mapping accuracy, respectively.

An experienced rater (author EM) used these reports to assess the mask's delineation of the brain boundary and the spatial transformation in reports for all images in the sample.

\section{RESULTS AND DISCUSSION}

\subsection{An atlas-based brain extraction tool for rodents adapted from human neuroimaging}

The proposed artsBrainExtraction workflow modifies the ANTs approach for non-human imaging. The workflow, presented in Figure 1, and registration configuration, which is available as a JavaScript Object Notation (JSON) file from nirodents/data, closely follows antsBrainExtraction.sh. However, the most fundamental change is the reference template; integration with TemplateFlow.org equips the workflow with programmatic access to a bank of population templates to choose from, such as Goerzen's Fischer344 template and binary brain mask [14], which artsBrainExtraction uses by default. Other adaptations to the ANTs' implementation include building the workflow with Nipype [15], and denoising the moving image using a non-local means filter [16] before it is corrected for signal inhomogeneity [13]. Further improvements include the com- 
patibility of parameter values for rodent images (e.g., the distance between B-Spline control points in the inhomogeneity correction step) and the implementation of select ANTs tools to ensure consistent orientation information is stored in the headers of intermediate and final images. For particularly challenging head positions, the original human workflow includes a step to initialize the nonlinear registration in a more robust manner. This step is disabled by default in artsBrainExtraction, because FOV prescription and head tilting are often more consistent between rodent images, due to fixation frames and specialized beds.

TemplateFlow integration provides great flexibility, as the workflow can be applied to any species with an available reference MRI template, and improves upon previous atlasbased brain extraction implementations for rodents, such as atlasBREX [6]. First, artsBrainExtraction does not require the creation of a study-specific template or the manual delineation of the brain on such a template. Second, the workflow has been designed (and evaluated) to perform correctly with templates from previous studies. Atlas-based methods are less reliant on prior information, and thus typically more robust, than their counterparts. For instance, methods that define the mask using image contrast may exclude regions with typically lower signal, such as the ventral surface of the brain [7]. Finally, existing methods (for both humans and rodents) generally require specific preprocessing steps and parameter adjustment [5, 7], but atlas-based brain extraction requires minimal user input.

An alternative approach to atlas-based brain extraction, using convolutional neural networks, appears close to a breakthrough. Although results are promising on human adults (e.g., github.com/neuronets/nobrainer,) the limited availability of annotated rodent MRI data required to train the models precludes its generalization across studies [3, 8].

\section{2. artsBrainExtraction performs reliably on multi-study data with varied imaging parameters}

All images in the composite dataset, which were largely diverse in terms of imaging protocols and parameters (see subsection 2.1), successfully passed through the tool pipeline. After screening, 98\% of the images (186) were rated as having a highly accurate brain mask, demonstrating the reliability against heterogeneous input data. In the 4 images that did not meet criteria, the mask did not adequately match the brain boundary in multiple slices, although the inaccuracies were not deemed deleterious so as to preclude further processing of these images. Two examples, showing a failing (panels AB) and a passing (panels C-D) image, are found in Figure 2. For transparency and scrutiny of the results, all reports are available at DOI: 10.17605/OSF.IO/7EJWV.

\section{CONCLUSION}

We introduce artsBrainExtraction for atlas-based brain extraction of rodent MRI, which emulates the ANTs tool for adult human brains and is robust to heterogeneous data inputs. This is the first tool from NiRodents, an open-source Python package available on GitHub (nipreps/nirodents), released under an Apache License 2.0, and distributed via Pypi or Linux containers. NiRodents intends to reliably adapt human-specific workflows for non-human data improving congruence between the fields.

\section{REFERENCES}

[1] S. M. Smith, "Fast robust automated brain extraction," Hum Brain Map, vol. 17, no. 3, pp. 143-155, 2002.

[2] F. Ségonne et al., "A hybrid approach to the skull stripping problem in MRI," NeuroImage, vol. 22, no. 3, pp. 1060-1075, 2004.

[3] N. Chou et al., "PCNN," IEEE Trans Imag Proc, vol. 20, no. 9, pp. 2554-2564, 2011.

[4] T. Wood et al., "rBET," in ISMRM, Salt Lake City, UT, USA, 2013, vol. 21, p. 2706.

[5] I. Oguz et al., "RATS," J Neurosci Meth, vol. 221, pp. 175-182, 2014.

[6] J. Lohmeier et al., "atlasBREX," Sci Rep, vol. 9, no. 1, pp. 12219, 2019.

[7] Y. Liu et al., "Automatic brain extraction for rodent MRI images," Neuroinf, vol. 18, no. 3, pp. 395-406, 2020.

[8] L.-M. Hsu et al., "Automatic skull stripping of rat and mouse brain MRI data using U-net," Front Neurosci, vol. 14, 2020.

[9] O. Esteban et al., "fMRIPrep," Nat Meth, vol. 16, no. 1, pp. 111-116, 2019.

[10] B. Avants et al., "Symmetric diffeomorphic image registration with cross-correlation," Med Image Anal, vol. 12, no. 1, pp. 26-41, 2008.

[11] R. M. Cohen et al., "A transgenic Alzheimer rat with plaques, tau pathology, behavioral impairment, oligomeric a $\beta$, and frank neuronal loss," J Neurosci, vol. 33, no. 15, pp. 6245-6256, 2013.

[12] N. Sirmpilatze et al., "Temporal stability of fMRI in medetomidine-anesthetized rats," Sci Rep, vol. 9, no. 1, pp. 1-13, 2019.

[13] N. J. Tustison et al., "N4ITK," IEEE Trans Med Imag, vol. 29, no. 6, pp. 1310-1320, 2010.

[14] D. Goerzen et al., "An MRI-derived neuroanatomical atlas of the Fischer 344 rat brain," Sci Rep, vol. 10, no. 1, pp. 1-13, 2020.

[15] K. Gorgolewski et al., "Nipype," Front Neuroinf, vol. 5, pp. 13, 2011.

[16] J. V. Manjón et al., "Adaptive non-local means denoising of MR images with spatially varying noise levels," $J$ Magn Reson Imaging, vol. 31, no. 1, pp. 192-203, 2010. 